\title{
Editorial
}

\section{The current status of myocardial disarray in hypertrophic cardiomyopathy}

\author{
M J DAVIES \\ From the British Heart Foundation Cardiovascular Pathology Unit, St George's Hospital Medical School, University \\ of London, London
}

When two eminent and experienced pathologists hold what appear to be diametrically opposed views on the specificity of the histological features of hypertrophic cardiomyopathy is is hardly surprising that clinicians are confused. This confusion has occurred as a result of the articles by Becker and Caruso ${ }^{1}$ and Maron. ${ }^{2}$ Stated simply the opposing views held by these workers are, on the one hand, that myocardial disarray is the pathognomonic feature of hypertrophic cardiomyopathy and, on the other, that since it can occur in normal hearts the specificity must be seriously questioned.

When such divergent views are held it is natural to look for further opinions from within the ranks of those concerned with tissue morphology who have equal experience of the condition in question. The hospital department in which Teare worked and from which came the first description ${ }^{3}$ of asymmetrical hypertrophy must surely fit this specification; moreover, the department has continued to see numerous cases both from hospital and forensic necropsies.

Teare originally stressed two salient features of hypertrophic cardiomyopathy ${ }^{3}$; firstly, the asymmetry of the septal hypertrophy and, secondly, the bizarre and disorganised arrangement of the muscle bundles in the ventricular septum. The absolute specificity of asymmetrical septal hypertrophy was questioned soon after his initial description, but only with the advent of echocardiography was it generally realised that only some patients with hypertrophic cardiomyopathy have disproportionate septal hypertrophy and conversely that a degree of asymmetry is found in severe left ventricular hypertrophy from whatever cause. In retrospect it is interesting that, of the eight original specimens from Teare's work extant, in only five is the left ventricle actually asym-

Requests for reprints to Professor M J Davies, Cardiovascular Pathology Unit, St George's Hospital Medical School, Cranmer Terrace, London SW17 ORE. metrical when measured. Teare could have added a feature, not recognised until years later by clinicians, that the disease process can be diffuse and produce symmetrical left ventricular hypertrophy.

Myocardial disorganisation at histological level, however, remained the "gold standard" for a diagnosis of hypertrophic cardiomyopathy and was further refined with a recognition that disorganisation could occur at three levels. Firstly, there is gross disorganisation of muscle bundles with interspersed bands of collagen responsible for the characteristic macroscopic whorled pattern of cut slices of the myocardium seen with the naked eye at necropsy. Secondly, there is the abnormality of myocardial cell to cell arrangement (disarray) visible by light microscopy. Foci occur in which myocardial fibres form complete circles or whorls around a central core of connective tissue. Finally, there is disorganisation of the myofibrillary arrangement actually within a single myocardial muscle cell, a feature detectable only by electron microscopy. It is with the appearances at light microscopy level that the current controversy is concerned. The myofibrillary disarray within individual cells is a constant feature of hypertrophic cardiomyopathy, but it does also occur frequently in many other conditions, particularly in those in which myocardial damage and associated hypertrophy have occurred.

At light microscopy level, beside the abnormalities of cell to cell arrangement (disarray), other features are present in hypertrophic cardiomyopathy. These include focal areas of very short broad, but normally arranged, muscle fibres with very large prominent hyperchromatic vesicular nuclei far greater in size than in ordinary hypertrophy. In addition, foci of connective tissue containing abundant mononuclear cells in the centre of whorls of myocardial cells may be found. While disarray is, therefore, not the only criterion available on which to make the histological diagnosis, it remains the feature that is regarded as vital. 
Maron, in a series of publications predominantly concerned with known clinical cases of hypertrophic cardiomyopathy, has championed the cause of the histological specificity of disarray. ${ }^{4-7} \mathrm{He}$ has, however, clearly recognised that disarray may occur to a limited degree in disorders other than hypertrophic cardiomyopathy. In postmortem hearts from patients clinically diagnosed as having hypertrophic cardiomyopathy assessment of the proportion of septal myocardium involved was about one third; in patients with other conditions producing hypertrophy and in normal hearts it was only approximately $1 \%$. Thus in Maron's view "disarray is a constant feature of hypertrophic cardiomyopathy" and when present in great degree it becomes specific.

Becker and Caruso were concerned with the absolute specificity of disarray as an isolated histological feature of hypertrophic cardiomyopathy. Thus they looked at normal hearts. Disarray could be found, and they concluded that it must be non-specific. For morphologists they also highlighted the fact that different planes of section can produce what appears to be disarray that vanishes in a plane cut at right angles. Others have also described a degree of disarray in various cardiac abnormalities including congenital heart disease. ${ }^{89}$ Thus in isolation disarray cannot be a totally specific histological feature of hypertrophic cardiomyopathy.

Where then does the truth lie? Both views are entirely correct in the context of the material to which they refer, and close reading of both papers shows discord between the protagonists only in what each thought the other meant in a wider context. The key concept to marry both views is that of a quantitative and not absolute specificity for disarray as an isolated histological feature.

What then are the practical consequences of the views of both groups being substantially correct? They are, firstly, that an experienced cardiac pathologist given the heart at necropsy from which he can take numerous sections will very rarely be in doubt about whether or not hypertrophic cardiomyopathy is present. This decision will be based on disarray being present, together with the other histological features, in at least $10 \%$ of the ventricular myocardium. Neither Maron nor Becker and Caruso would contest this view.

Secondly, it is now a foolhardy pathologist who makes a dogmatic diagnosis of hypertrophic cardiomyopathy based on biopsy material, a mistake that many of us made in the early heady days of cardiac biopsy. The whole aim of Becker and Caruso's work was to establish this fact, and there is nothing in Maron's writings to contradict this view. There is too great a chance of an area of disarray being fortuitously taken from a normal heart. It is equally true that one random block of myocardium taken at necropsy will not and cannot confirm or refute a diagnosis of hypertrophic cardiomyopathy. Too many pathologists still believe it will, and it is here that the value of the work of Becker and Caruso lies. In essence their paper is not describing how to diagnose hypertrophic cardiomyopathy by its histological characteristics but is indicating how to avoid its overdiagnosis. The misconception had undoubtedly been growing among pathologists with limited experience of cardiac morphology that even a single focus of disarray was diagnostic. This misconception derived originally from an otherwise excellent description of the morphology of hypertrophic cardiomyopathy, ${ }^{10}$ in which the word "unique" was applied to disarray. It is clear that the plane of histological section in a single block can produce spurious appearances of disarray though without the other subsidiary features. The more blocks that are taken and the more that contain disarray the more certain will be the result.

There do remain uncertainities as a result of the work of both of these groups. Since the presence of disarray is quantitative rather than absolute there will be cases on the borderline of normality. Personal experience suggests these to be very rare indeed, but in exceptional cases final judgment has to be made on a combination of clinical features and histology.

A second as yet unresolved difficulty occurs with a small group of patients who have been clinically diagnosed as having hypertrophic cardiomyopathy but who do not have disarray. There are two possibilities; they may represent a different, totally separate form of hypertrophic cardiac muscle disease or they may be a variant of true "Teare's" disease. If the latter is confirmed by family studies in which there are cases with and without disarray this will be an indication that disarray not only has no specificity but also no functional significance. Until such a family can be described disarray - when present to more than trivial degrees-must remain as the morphological manifestation, with considerable pathophysiological significance, for the genetically transmitted myocardial defect which is known as hypertrophic cardiomyopathy.

\section{References}

1 Becker AE, Caruso G. Myocardial disarray. A critical review. Br Heart f 1982; 47: 527-38.

2 Maron BJ. Myocardial disorganisation in hypertrophic cardiomyopathy. Another point of view [Editorial]. $\mathrm{Br}$ Heart f 1983; 50: 1-3.

3 Teare D. Asymmetrical hypertrophy of the heart in young adults. $\mathrm{Br}$ Heart $\mathcal{f}$ 1958; 20: 1-8.

4 Maron BJ, Roberts WC. Quantitative analysis of cardiac muscle cell disorganization in the ventricular septum of 
patients with hypertrophic cardiomyopathy. Circulation 1979; 59: 689-706.

5 Maron BJ, Sato N, Roberts WC, Edwards JE, Chandra RS. Quantitative analysis of cardiac muscle cell disorganisation in the ventricular septum: comparison of fetuses and infants with and without congenital heart disease and patients with hypertrophic cardiomyopathy. Circulation 1979; 60: 685-96.

6 Maron BJ, Anan TK, Roberts WC. Quantitative analysis of the distribution of cardiac muscle cell disorganization in the left ventricular wall of patients with hypertrophic cardiomyopathy. Circulation 1981; 63: 882-94.

7 Maron BJ, Roberts WC. Hypertrophic cardiomyopathy and cardiac muscle cell disorganization revisited: relation between the two and significance. Am Heart $\mathcal{F} 1981 ; 102$ : 95-110.

8 Van Der Bel-Kahn J. Muscle fibre disarray in common heart diseases. Am F Cardiol 1977; 40: 355-64.

9 Bulkley BH, Weisfeldt ML, Hutchins GM. Asymmetric septal hypertrophy and myocardial fibre disarray: features of normal, developing, and malformed hearts. Circulation 1977; 56: 292-8.

10 Ferrans VJ, Morrow AG, Roberts WC. Myocardial ultrastructure in idiopathic hypertrophic subaortic stenosis. A study of operatively excised left ventricular outflow tract muscle in 14 patients. Circulation 1972; 45: 769-92. 